\title{
SPH MODELING OF MEAN VELOCITY CIRCULATION IN A RIP CURRENT SYSTEM
}

\author{
ROZITA JALALI FARAHANI ${ }^{1}$, ROBERT A. DALRYMPLE², ALEXIS HÉRAULT ${ }^{3}$, GIUSEPPE \\ BILOTTA $^{4}$
}

\begin{abstract}
A Lagrangian numerical model called Smoothed Particle Hydrodynamics is used to analyze rip current system generated by a single bar and a rip channel. The pattern of the wave-induced circulation cell over the bar, the oppositely-rotating circulation cell on-shore and a strong seaward-directed current in the rip channel is modeled numerically. The mean horizontal variations of rip current system as well as three-dimensional circulations are studied. The results in three-dimensional space reveal the wave-current interaction and flow patterns in different parts of rip channel, bar, and the trough located near shore. For comparison to experimental data, Eulerian nodes are introduced to the numerical model and SPH interpolation over neighboring Lagrangian particles is implemented to find fluid parameters at those specific nodes. This methodology leads to a better understanding of depth-integrated flows and a more accurate comparison of numerical results with experimental results. Model predictions are compared to laboratory measurements of Drønen et al. (2002) and show good agreement, including mean velocity profiles, mean surface elevation and three-dimensional velocity components.
\end{abstract}

Keywords: rip current system; Smoothed Particle Hydrodynamics; near-shore circulations

\section{Introduction}

Rip current systems consist of primary offshore directed water flows that begin in the surf zone and the associated wave-induced circulation. Incident waves carry mass, energy and momentum. At the breaker line, waves begin dissipating energy and losing momentum. To balance the decrease in onshore wave momentum, a hydrodynamic force is generated, which leads to the increase of the mean water level at the shoreline (set-up). A variation of wave height along the shore leads to the variation of setup, which results in rip currents. Different types of rip currents are caused by various mechanisms. These mechanisms can be classified as: 1. Bathymetric variations, 2. Wave-Wave interactions and 3. Alongshore currents and shear instabilities (See the recent review paper by Dalrymple et al. (2011)). Shepard et al. (1941) discussed that the height and trend of the waves are important factors in the development of rip currents. Coastal and shoreline configuration and tide were among the other factors, which were considered to be influential to the generation of rip currents. Later Mckenzie (1958) pointed out that the slope and regularity of the near-shore sea floor are factors that affect the way in which the waves are broken and refracted. Bowen and Inman (1969) first developed a theoretical derivation of rip currents using the equations of momentum and continuity including radiation stresses in shallow water. Then they studied rip currents induced by the interaction of edge waves and incident waves. These rip currents were caused by wave interaction, rather than topography variations. A set of experiments were performed in a $50 \mathrm{ft}$. by $60 \mathrm{ft}$. wave basin and edge waves generated by incident waves were observed and the relationship between rip currents and edge waves was investigated. Rip currents occurred at alternate antinodes of the edge waves. Spacing between the rip currents was equal to the longshore edge wavelength.

Rip currents resulting from bathymetric variations have been studied in various field measurements, laboratory experiments and numerical models. Wright and Short (1984) observed a quasi-systematic morphodynamic in the surf zone, studying 4 years of data from the coast of Australia. Haller et al. (1997) studied rip current systems, which are related to alongshore variations of underlying bed topography. They carried out a series of experiments in a $17 \mathrm{~m}$ by $18 \mathrm{~m}$ wave basin with three fixed sand bars with two rip channels between them. Interaction of the surface waves with rip currents and the nearshore circulation generated by wave breaking on a barred beach were investigated. Their measurements included depth-integrated velocities, and were later modeled numerically by Chen et al. (1999), who used Boussinesq equations to model rip current as a part of nearshore circulation. They studied the spatial and temporal variability of nearshore circulations and compared their results with Haller experiments. Hass et al. (2002), (2003) conducted a laboratory measurement of the vertical structure of rip currents using the bed topography of Haller et al. (1997). Then they numerically

\footnotetext{
${ }^{1}$ Civil Engineering Department, Johns Hopkins University, Baltimore, MD, USA, rozita@jhu.edu

${ }^{2}$ Civil Engineering Department, Johns Hopkins University, Baltimore, MD, USA, rad@jhu.edu

${ }^{3}$ Conservatoire National des Arts et Métier, Paris, France, alexis.herault@cnam.fr

${ }^{4}$ Università degli studi di Catania, Catania, Italia, bilotta@dmi.unict.it
} 
modeled the experimental results by the use of a quasi-three-dimensional numerical model. In their numerical model, the depth-integrated wave-averaged equations are solved and the effect of the vertical variation of the currents is separately included in the depth-integrated model. Drønen et al. (2002) performed a laboratory study of flow over a single bar and a rip channel. The experiments were performed in a $4 \mathrm{~m}$ by $30 \mathrm{~m}$ wave tank, which represents one-half of a rip current system, represented by two bars with a rip in between (using symmetry about the sidewalls). The two shore-normal sidewalls of this experiment correspond to the centerline of rip channel and centerline of the bar, located between two rip channels, respectively. They measured 3D velocity profiles and mean water levels. Particle trajectories tests were also performed to track the flow in a rip current system.

In the present study, Smoothed Particle Hydrodynamics (SPH) is used to study the threedimensional structure of the rip current and nearshore circulation, generated by longshore variation of wave height due to the submerged bar. Three-dimensional equations of motion and continuity are solved and $3 \mathrm{D}$ variations of flow due to wave-current interaction as well as depth-integrated mean patterns are studied.

$\mathrm{SPH}$ is a robust numerical method that is capable of modeling flows with complicated deformations, including breaking waves. The computational domain is represented by discrete particles that are carrying fluid parameters/variables and no predefined connectivity is considered for these particles. Monaghan (1994) first modeled free surface problems using SPH and based on Navier-Stokes equations that were discretized by a kernel function. He implemented SPH to model a bursting dam and a wavemaker, generating waves breaking on a beach. Later SPH was used to model a wide range of problems such as modeling of landslide-generated waves (Panizzo et al., 2004), impact of wave on structures (Dalrymple et al., 2002) and simulation of swimming linked bodes (Kajtar et al., 2008)

Most numerical models, which were used previously to simulate rip current systems and waveinduced nearshore circulations, are based on depth-integrated, time-averaged governing equations of conservation laws of mass and momentum. However experimental measurements reveal that the threedimensional structure of flow plays an important role in the formation of flow patterns in the rip current system, which cannot be completely captured in two-dimensional depth-integrated viewpoint. In this study SPH is employed to investigate wave breaking-induced circulations and three-dimensional interaction between wave and rip current. In addition, in order to compare the two-dimensional depthintegrated mean flows with experimental results, Eulerian fixed positions are set in the numerical model and fluid variables at those fixed nodes are calculated using SPH interpolation. The final model is capable of finding fluid variables on Eulerian fixed nodes as well as Lagrangian moving particles and comparison of both two-dimensional mean patterns and three-dimensional variations of flow are possible. Results are compared to the laboratory measurements of Drønen et al. (2002), including mean horizontal velocities, mean water level, longshore and cross-shore currents and three-dimensional structure of flows.

\section{SPH Governing Equations}

The governing equations are the Navier-Stokes equations, which are discretized using interpolation kernel functions that approximate a delta function. The interpolation is applied on a set of moving particles. Each particle carries fluid variable such as mass, velocity and pressure. The value of any function as $A(\vec{r})$ at a particle can be approximated by summing over the properties of its neighboring particles. SPH interpolation of function $A(\vec{r})$ and its gradient can be written as (Monaghan and Kos, 1999):

$$
\begin{aligned}
A(\vec{r}) & =\sum_{j} m_{j} \frac{A_{j}\left(r_{j}\right)}{\rho_{j}} W\left(\left|\vec{r}-\vec{r}_{j}\right|, h\right) \\
\nabla A(\vec{r}) & =\sum_{j} m_{j} \frac{A_{j}\left(r_{j}\right)}{\rho_{j}} \nabla W\left(\left|\vec{r}-\vec{r}_{j}\right|, h\right)
\end{aligned}
$$


where particle $j$ corresponds to the neighboring particle; $\rho=$ particle density; $m=$ particle mass; $W=$ interpolation kernel function and $h=$ Smoothing length of kernel function.

The continuity equation can be written as:

$$
\frac{D \rho}{D t}=-\rho(\nabla \cdot \vec{u})
$$

where $\rho=$ density, $t=$ time, and $\vec{u}=$ particle velocity. The SPH representation of continuity equation is:

$$
\frac{D \rho_{i}}{D t}=\sum_{j} m_{j}\left(\vec{u}_{i}-\vec{u}_{j}\right) \cdot \nabla_{i} W_{i j}
$$

where $i$ corresponds the particle of interest and $j$ corresponds to the neighboring particles.

Conservation of momentum equation is written as follows:

$$
\frac{D \vec{u}}{D t}=-\frac{\nabla P}{\rho}-\nabla \phi+\vec{g}
$$

where $P=$ particle pressure and $\phi=$ viscous stress tensor. The SPH representation of momentum equation is:

$$
\frac{D \vec{u}_{i}}{D t}=-\sum_{j} m_{j}\left(\frac{p_{i}}{\rho_{i}^{2}}+\frac{p_{j}}{\rho_{j}^{2}}+\Pi_{i j}\right) \nabla_{i} W_{i j}+\vec{g}
$$

The term $\Pi_{i j}$ represents the viscosity term. In this study a Sub-particle scaling (SPS) term, which was introduced by Dalrymple and Rogers (2005), is used to capture the turbulence occurred in breaking waves. Large eddies are taking in to account by SPS stress tensor, while small eddies are modeled by Smagorinsky eddy viscosity model with a constant coefficient.

In this model the fluid is assumed to be a weakly compressible fluid, which has a very small density fluctuations. This is satisfied by the fact that the speed of sound is chosen to be much larger (by at least 10 times) than the largest velocity in the model. Pressure at each time step is calculated using the equation of state as:

$$
P=\beta\left(\left(\frac{\rho}{\rho_{0}}\right)^{\gamma}-1\right)
$$

where $\gamma=7$ and coefficient $\beta$ can be considered as:

$$
\beta=\frac{\rho_{0} c^{2}}{\gamma}
$$

Different methods for modeling the fixed or moving boundaries are introduced. In our study we used repulsive normal forces that were first introduced by Monaghan (1994). These forces provide the pressure, which prevents fluid particles to penetrate into boundary particles. Repulsive forces were chosen to be similar to Lennard-Jones forces of molecular dynamics. Later Monaghan and Kajtar (2009) introduced a new form of boundary forces. The boundary particles should have a smaller particle spacing of ratio $1 / 2$ in comparison to fluid particles. The force per mass of fluid particle $i$ due to boundary particle $b$ can be given as:

$$
\vec{f}_{i b}=\frac{k}{\beta} \frac{\vec{r}_{i b}}{r_{i b}^{2}} W_{i b} \frac{2 m_{b}}{m_{i}+m_{b}}
$$


Coefficient $k$ is considered to be equal to $g D$ where $D$ is the depth of fluid. Coefficient $\beta$ ensures that the variation of boundary particles spacing doesn't affect the fluid particle force. The momentum equation considering the boundary conditions will be in the form of:

$$
\frac{D \vec{u}_{i}}{D t}=-\sum_{f} m_{f}\left(\frac{p_{i}}{\rho_{i}^{2}}+\frac{p_{f}}{\rho_{f}^{2}}+\Pi_{i f}\right) \nabla_{i} W_{i f}+\vec{g}+\sum_{b}\left[\vec{f}_{i b}-m_{b} \Pi_{i b} \nabla_{i} W_{i b}\right]
$$

where here, $f$ corresponds to fluid particles and $b$ corresponds to boundary particles. In this study the recent radial forces have been implemented and the mass of fluid particles and boundary particles are considered to be equal.

\section{GPU-SPH}

SPH is computationally intensive for large and complicated cases. This issue leads to the use of parallel computing, which has mostly been done on multiple Central Processing Units (CPUs) of computers. Recently advances in computer technology permit the use of Graphical Processing Units (GPUs) for parallel computing with high computational power and low expenses. GPU is massively parallel and on the other hand, the data-parallel nature of SPH method makes it perform well on multithreaded GPU. GPU programming can be traced back to General-Purpose computation on GPU (GPGPU). This older method required programmers to understand graphic programming languages in order to do numerical computations. Recently a new GPU parallel architecture is introduced as Compute Unified Device Architecture (CUDA), which uses high-level languages such as $\mathrm{C}, \mathrm{C}++$ and Fortran to program the graphic cards so computation on the GPU has become easier.

In this study the GPUSPH free surface flow SPH package developed by Hérault et al. (2010) is used. The program consists of two types of codes: The ones that are run on CPU and the ones that are run on GPU. Initial data and the problem configuration are first carried out on the CPU and then the essential data are transferred to GPU where the heavy-duty computation takes place. SPH calculations, including the search for neighboring particles, the calculation of forces using the governing equations and the calculation of pressure, velocity and position of particles for the next time step, are performed on graphic card. Then the results are transferred to CPU to be recorded as output files or to be displayed as frequently as desired.

Since the interpolation kernel has compact support, not all particles contribute to the sums in the governing equations, just the neighboring particles. Neighboring particles search is implemented using a sorting algorithm that optimizes the searching distances and satisfies the coherence of graphic card texture. In this algorithm, a uniform grid is implied to the computational domain and each particle is assigned to a grid cell according to its center point. Then particles are reordered and sorted based on their cell situation. When the sorted list is created, searching for neighboring particles of each particle takes place only in the cells around the particle cell as well as the particle cell itself. Sorting particles helps to improve the texture memory coherence and decreases the lookup distances so the searching time decreases respectively. GPUSPH is reported (Hérault et al. (2010)) to have a one to two orders of magnitude faster computational time than the equivalent CPU code and it can be run on a single or multiple Nvidia GPU cards.

\section{Rip current system}

In order to model a rip current system caused by bathymetric variations, an idealized bathymetry consists of a single bar and a rip channel is considered. The bathymetry was inspired by the wave tank experiment of Drønen et al. (2002), which corresponds to one half of a wavelength of a bar/rip channel system. In the experiment, one shore-normal wall of the tank corresponds to the symmetry line of rip channel normal to the beach and the other shore-normal side wall of the tank corresponds to the symmetry line of the bar between two rip channels. In fact, this bathymetry can be interpreted as a section of a multiple rip current system that consists of along-shore sand bars, periodically intersected with rip channels in along-shore direction. The three-dimensional structure of wave-induced rip current and surf zone circulations as well as mean currents were observed in this set of experiments. Therefore, the numerical studies will be compared with both three-dimensional structures of rip current as well as two-dimensional depth-integrated mean flows. This experiment was performed in a $4 \mathrm{~m}$ wide and $30 \mathrm{~m}$ long wave tank. The bar is $4.8 \mathrm{~m}$ long, $3 \mathrm{~m}$ wide and $0.13 \mathrm{~m}$ high and has a slope of $1 / 27$ in crossshore direction. The width of the rip channel is $1 \mathrm{~m}$ and has the same slope of $1 / 27$. The beach has a slope of 1/17. Figure (1) illustrates the bed topography of the Drønen experiment. 


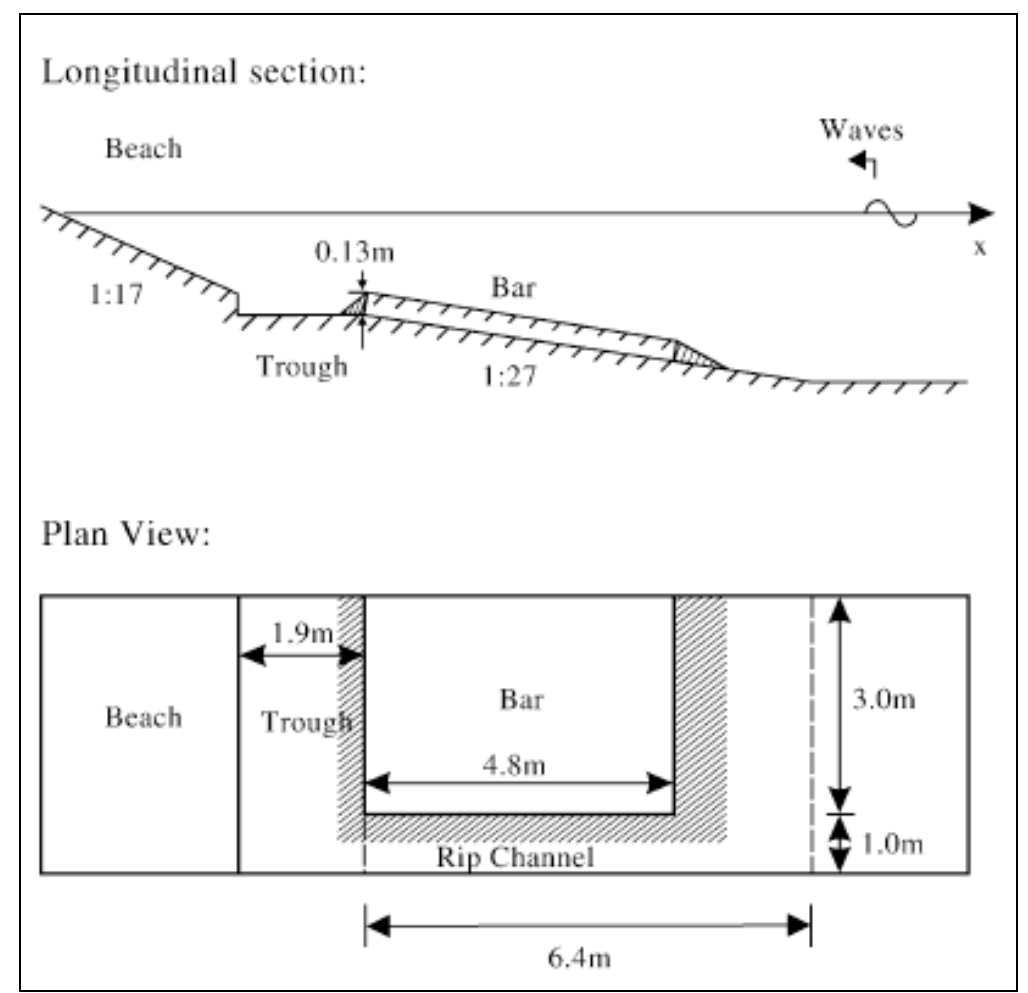

Figure 1. Rip current system bathymetry. Top: Side view, Bottom: Plan view (Drønen et al., 2002)

In our model, the still water depth at the bar crest is $0.15 \mathrm{~m}$, wave height is $0.19 \mathrm{~m}$ and wave period is equal to $1.5 \mathrm{~s}$. This idealized bathymetry is modeled using GPUSPH model using about three million particles and particle spacing is equal to $0.02 \mathrm{~m}$. A piston wave maker is considered as the wave generator that produces regular waves normal to the coastline and is located around $6 \mathrm{~m}$ from the beginning of the sloping part of the bed topography (around $12.4 \mathrm{~m}$ far from the crest of the bar). The Wendland kernel function is used as the weighting function in SPH interpolation and Shepard filtering is implied every 20 time steps in order to reinitialize particle densities for smoother results. Figure (2) shows the bed bathymetry, which is applied to the numerical model where the wave maker is in the right hand side and beach is located on the left hand side.

\section{Fluid parameters on Eulerian nodes}

The Lagrangian nature of Smoothed Particle Hydrodynamics makes it capable of modeling complicated fluid problems with large deformations. However, post-processing flow analysis based on SPH output can be problematic since data are known on irregularly-spaced particles and it is difficult to compare the results with experimental results that are measured at fixed positions. In order to solve this problem and compare the data computed on Lagrangian moving particles with the data measured on Eulerian fixed nodes, a straightforward method is used to interpolate scattered data to a regular grid. For this purpose, a Cartesian grid, which covers the entire computational domain, is introduced to the model. For each node of this grid, neighboring moving particles are found and the fluid parameters of the node is calculated using the fluid parameters of its neighboring moving particles as follow:

$$
A_{n}=\sum_{p} m_{p} \frac{A_{p}}{\rho_{p}} W\left(\left|r_{n}-r_{p}\right|, h\right)
$$

where notation $n$ corresponds to a fixed node, notation $p$ corresponds to the moving particles and $A$ can be any fluid parameter such as velocity, pressure or density. Figure (3) shows the interaction of fixed nodes and moving particles. SPH interpolation of particle data onto a regular Cartesian grid is implemented in GPUSPH package as fixed nodes called TESTPOINTS. TESTPOINTS can act as gages from which velocity, pressure and other flow quantities can be measured at specific locations. 


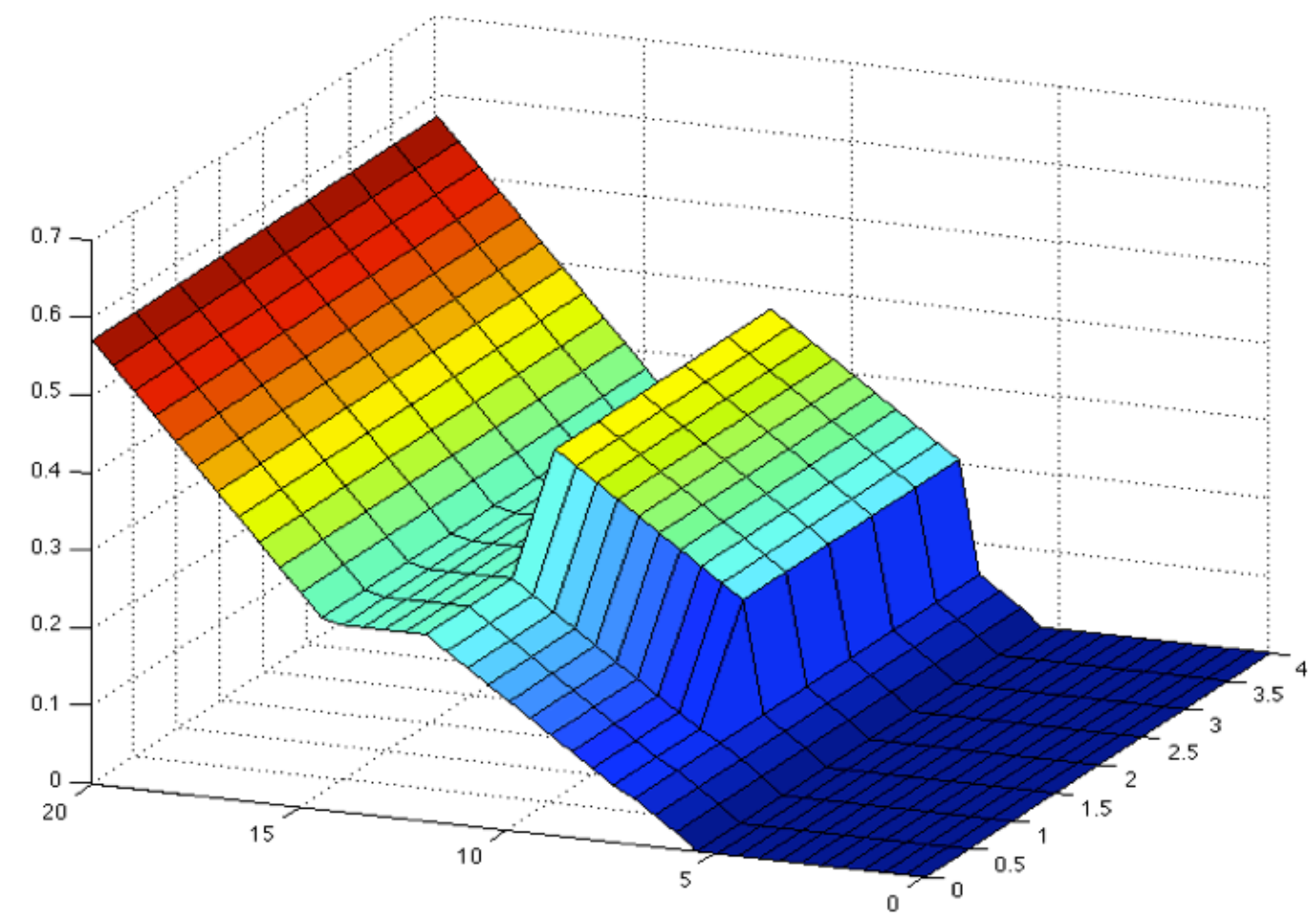

Figure 2. Bottom bathymetry applied to the numerical model

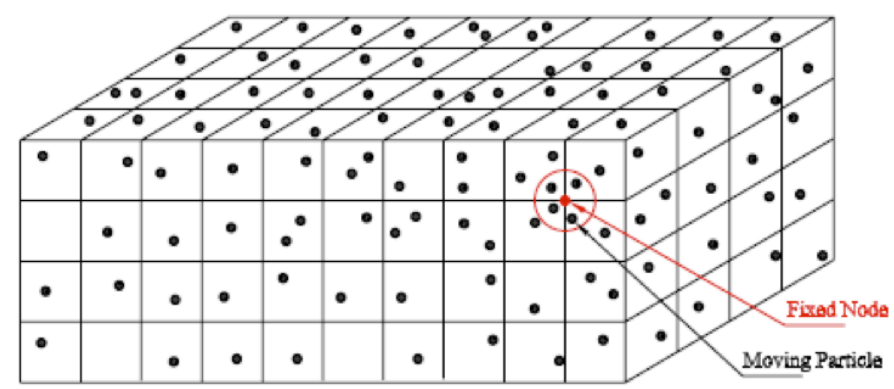

Figure 3. Schematic computational domain and position of fixed nodes and moving particles

Mapping fluid information from scattered particles into Eulerian grid nodes is used to calculate depth-averaged circulations. In addition, it improves post-processing analysis since it gets easier to study iso-surfaces, contour plots and slices. Since TESTPOINTS are used for analysis and comparison of SPH numerical data with experimental data, the mapping can be applied only periodically (typically every 10 time steps when output data is saved) so it doesn't impose an appreciable increase to the computational time.

\section{Free-surface particles detection}

A free-surface detection algorithm is used to find the free surface particles. The algorithm is based on the SPH kernel interpolation in order to be compatible with the SPH computation of governing equations. Thus this method is easy to implement and has a cheap computational expense since it is using the existing neighboring lists of particles and the same kernel function for interpolation. The freesurface tracking technique is similar to the algorithm proposed by Marrone et al. (2010) and is used at each time step of numerical simulation without a significant increase of the computational time. 
Free-surface detection algorithm consists of two steps: In the first step, a local normal vector for each particle is computed using gradient of kernel function. The normal vector for particle $i$ and components of kernel gradient can be defined as:

$$
\begin{gathered}
\vec{n}_{i}=\frac{\vec{v}_{i}}{\left|\vec{v}_{i}\right|} \\
\vec{v}_{i}=\sum_{j} \nabla W_{i j} V_{j}=\left\{\begin{array}{l}
\sum \frac{m_{j}}{\rho_{j}} \frac{d W}{d r} \frac{1}{r}\left(\vec{x}_{j}-\vec{x}_{i}\right) \\
\sum \frac{m_{j}}{\rho_{j}} \frac{d W}{d r} \frac{1}{r}\left(\vec{y}_{j}-\vec{y}_{i}\right) \\
\sum \frac{m_{j}}{\rho_{j}} \frac{d W}{d r} \frac{1}{r}\left(\vec{z}_{j}-\vec{z}_{i}\right)
\end{array}\right.
\end{gathered}
$$

where $\vec{n}$ is normal vector, $i$ corresponds to the particle of interest and $j$ corresponds to the neighboring particle.

In the second step, geometrical properties of free surface particles are used for their accurate detection. For each particle a cone is defined with particle's normal vector as its axis and a cone angle that has been set to be equal to $\pi / 6$. Then a control check is applied to find whether or not at least one neighboring particle exists in this cone region. If no neighboring particle is found, then that particle belongs to the set of surface particles. This control condition is given as:

$$
\left[\forall j, \frac{\vec{n}_{i} \cdot \vec{r}_{i j}}{r} \succ \cos (\text { cone-angle }) \Rightarrow i \in \text { Free-Surface-Particles }\right]
$$

Figure (4) shows a schematic figure of free surface detection. In order to have more accurate detection, cone angle can be varied according to the position of the particle. For instance for the particles that are close to the boundary particles, a larger cone angle is used to avoid misdetection.

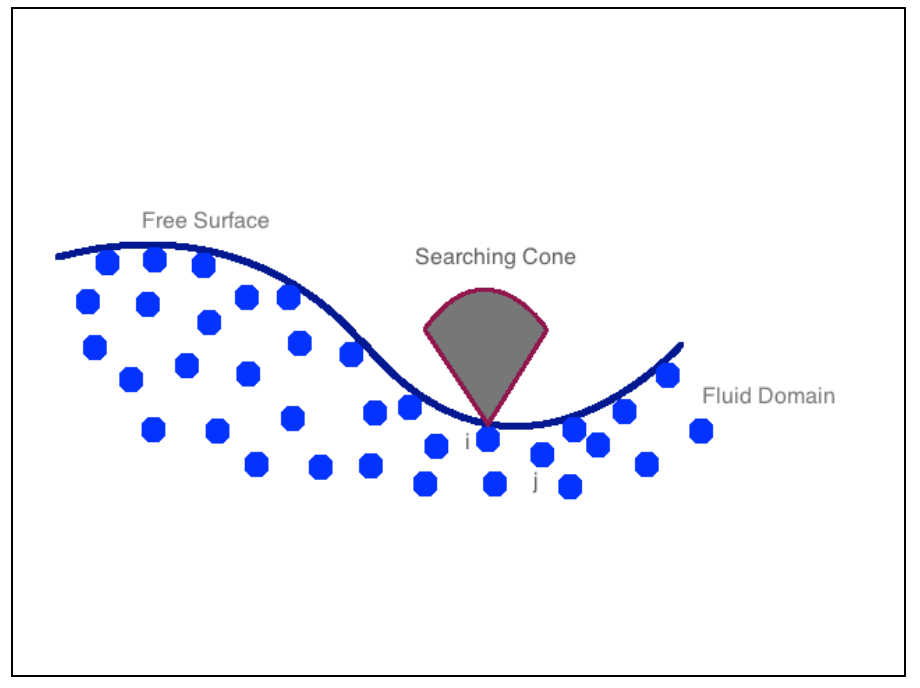

Figure 4. Schematic figure of free surface detection

Free surface GPU-kernel is composed of two loops over neighboring particles. In the first loop local normal vectors are computed and in the second loop cone condition is checked to find whether or not the particle is on the free surface. Since we are using the existing neighboring list of particles, this algorithm doesn't impose a significant increase to the computational cost. For the problems where 
planes are used as boundary condition, the fluid particles close to the planes may be misdetected as surface particles. The reason is that planes do not have any boundary particles and they just imply repulsive forces to the fluid particles close to them. So the fluid particles at their neighborhood may not find any particle in their cone region. To fix this problem, local normal vectors for the fluid particles close to planes are modified using the normal vectors of the planes. After this modification the normal vectors for these specific particles will be directed inward to the fluid so the problem will be solved.

Free surface detection has been tested for a couple of GPUSPH test cases (Rip current problem, dam break problem with planes as boundary conditions, open channel problem with periodic boundary condition...) and satisfactory results were observed.

\section{Numerical results and comparison with experimental data}

\section{Mean horizontal circulations}

The numerical model provides free surface elevations and velocity components for wave-current interaction motion on moving particles as well as fixed positions. Different flow quantities such as mean water level, three-dimensional structure of underlying current field, tracking the trajectory of water particles, and the vorticity pattern corresponding to the wave-induced surf zone circulation can be derived from the numerical results. The results are studied after the wave generator was running for at least 10 periods to eliminate start-up transient errors.

Figure (5) shows a snapshot of numerical results. This figure illustrates a side view (top figure) and a plan view (bottom figure) of rip current system with a bar at almost middle left and a rip channel at middle right of the wave tank. Wave generator is located at the right hand side of the wave tank. Several interesting phenomena are observed from this figure:

1. Wave shoaling over the sloping bar

2. Wave breaking over the submerged bar

3. Wave-current interaction in the rip channel

4. Wave diffraction due to rip current

5. Wave refraction due to inhomogeneous media

6. Higher harmonics generation (Mei and Ülüata, 1972) over the submerged bar

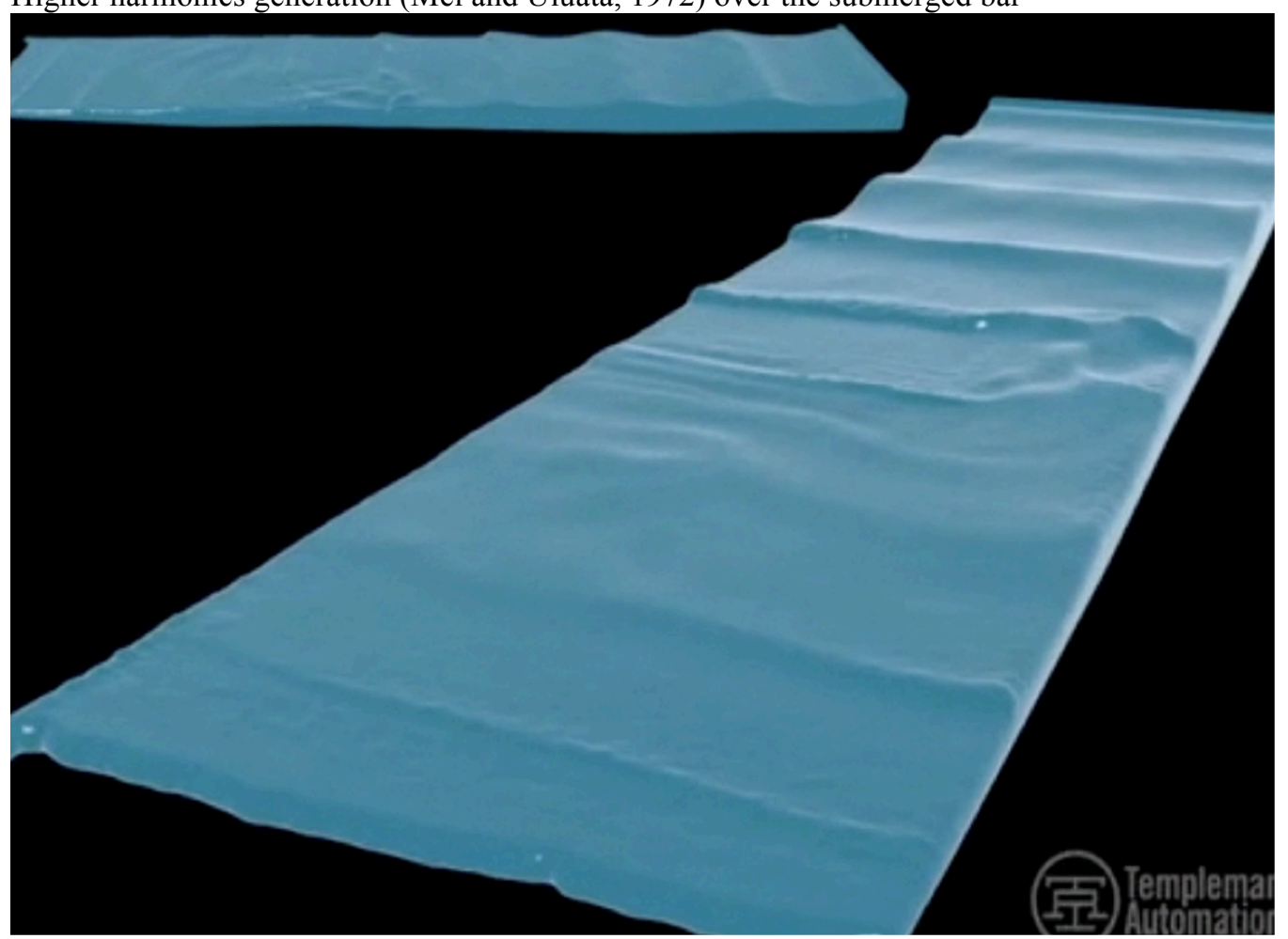

Figure 5. SPH numerical modeling of bar/rip channel system

Waves travel from the wave generator with nearly uniform crests until they reach the bar. On the sloping bar, wave shoaling and subsequent wave breaking is captured while there is almost no breaking in the rip channel. When the waves break, the radiation stress, which is the excess flow of momentum 
due to the presence of the waves, transfers to the water column and forces a mean water level increase. Therefore the mean water level on the bar is higher than the mean water level in the rip channel and this gradient in setup drives the current from the bar toward the channel. Longshore currents flowing from the bar into the channel act as feeders for the rip current flow, which travels in cross-shore direction towards the sea. In the rip channel, the underlying current affects the surface wave transformation and leads to wave-current interaction. The numerical results predict longshore variation of wave height due to the wave refraction by the rip current. The development of rip current and circulation cells causes the wave crest to be refracted as captured in the figure. When the waves propagate over the inhomogeneous media and pass the submerged bar, due to nonlinearity, higher harmonics are generated and secondary wave crests appear in the surf zone.

A snapshot of velocity profile (plan view), after $40 \mathrm{~s}$ of simulation time has passed, (after about 27 waves) is given in Figure (6).

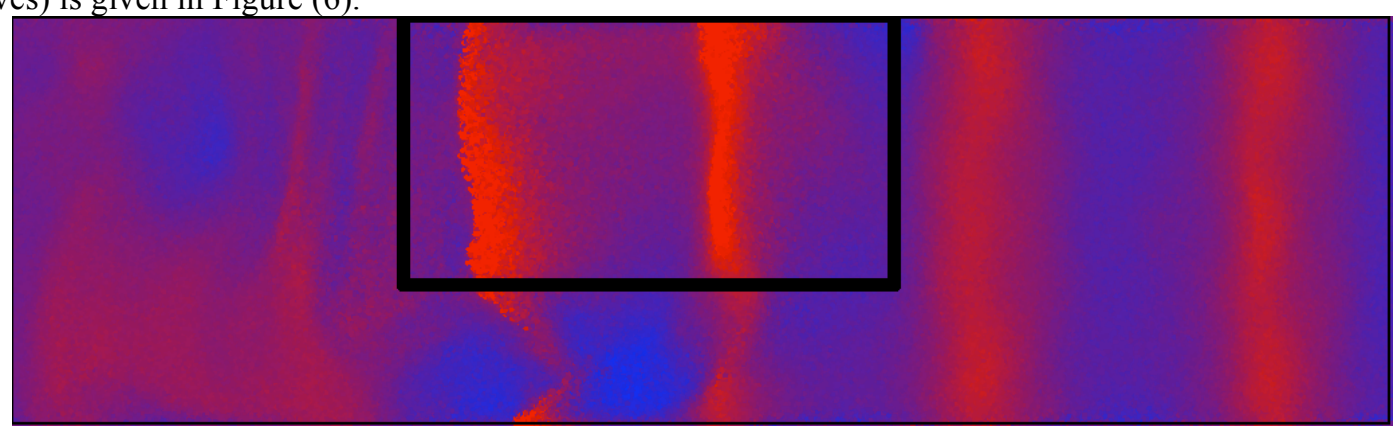

Figure 6. Plan view snapshot of flow over the bar and in rip channel in Time $=40-\mathrm{s}$

Waves are traveling from the right hand side of the picture and red color corresponds to higher velocity. Breaking waves are shown over the submerged bar. As it can be seen in the figure, there is a nonuniform pattern of breaking waves on the bar that leads to differences in set-up and produces the cell circulation over the bar, which will be discussed later. Waves seem to break more at the end of the bar (left hand side) so the set-up in this region becomes the main feeder of rip current in the channel. To find the horizontal mean velocity circulation, velocities are obtained at fixed, equally distributed positions (the Eulerian grid) over the water depth using the SPH interpolation over neighboring moving particles. Then the velocities at the fixed nodes are integrated over the depth and also averaged over one wave period. Mean horizontal velocity distribution in both numerical model and experimental model is given in figure (7).
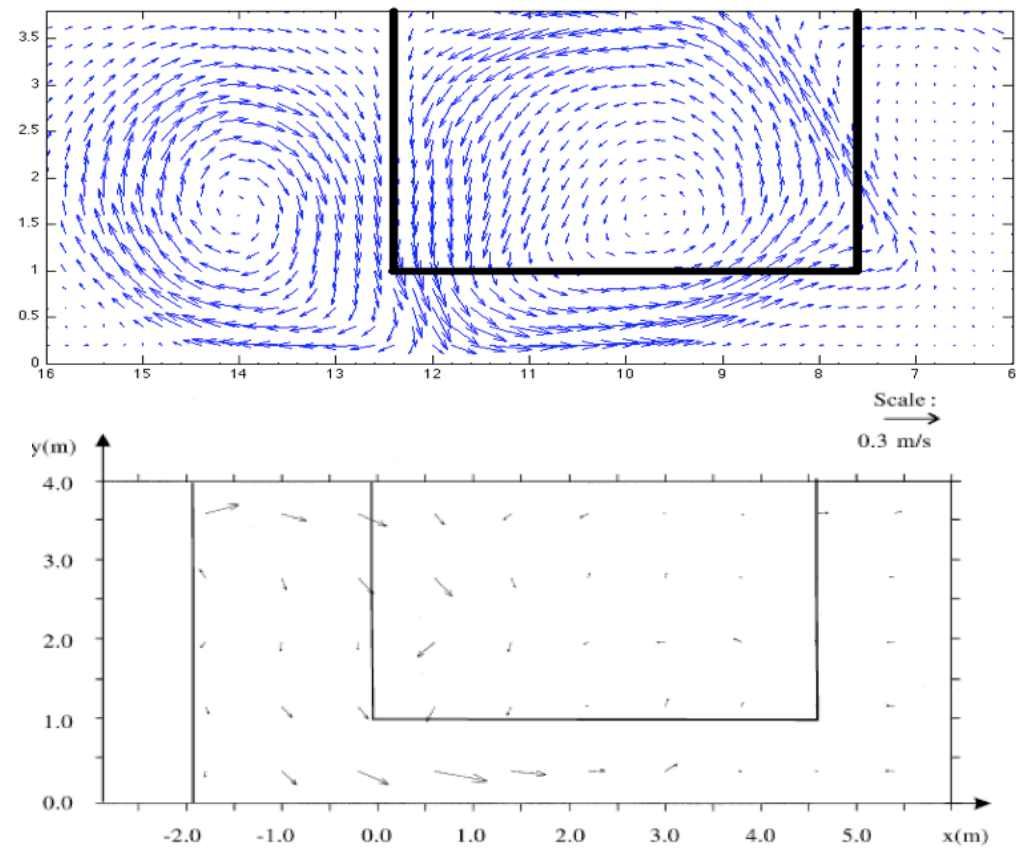

Figure 7. Mean horizontal velocity distribution. Top: Numerical results. Bottom: Experimental result 
The flow pattern predicted by numerical model is similar to the measured velocity field. In this picture, wave generator is located outside of the figure to the right hand side and waves are traveling from right to the left. In the Drønen experiment, the velocity, measured at one third of the depth, is considered to be the mean velocity but in numerical simulation, the actual mean velocity is obtained by taking the average of velocity over the water depth. The rip current system consists of an offshoredirected rip current in the channel, a circulation cell over the bar and an onshore oppositely-rotating circulation cell, can be observed from numerical results.

The circulation cell over the bar appears due to differences in set-up over the bar and in the rip channel. A second cell rotating in the opposite direction of the first circulation cell is observed in the surf zone. As the waves travel toward the shore, they break over the submerged bar, while there is almost no breaking over the rip channel. After the bar, in the surf zone, waves behind the channel are larger since they haven't been broken yet but the waves traveling over the bar are smaller since they have already been broken over the bar. The larger waves behind the channel lead to more intense breaking in the surf zone, creating another setup gradient, this time in the opposite direction of the setup gradient over the bar. Therefore a secondary opposite-rotating circulation occurs in the nearshore area. The second cell on the beach is also captured during the dye injections observations of Drønen et al. experiments. The rip current at the end of channel tends to move back onto the bar and join in the cell circulation over the bar region. This tendency has been also observed in experimental results (Drønen, et al. 2002).

Mean surface elevation is also measured numerically over one wave period, after $40 \mathrm{~s}$ have elapsed in the simulation time and the results are compared with the experimental data. Mean surface gradients result in pressure gradients providing the driving force for rip currents in bar/rip channel systems as was discussed by Dalrymple et. al (2011). In addition, cross-shore variation of setup also exists due to the non-uniform pattern of breaking waves. The differences in the wave breaking locations lead to a cross-shore gradient of mean free surface, which results to the circulations. Figure (8) illustrates the mean surface water elevation and corresponding gradients. On the left hand of the rip channel where rip current is entering the rip channel, the surface elevation is higher than the surface elevation on the bar crest due to the wave-current interaction and the absence of wave breaking in the channel.
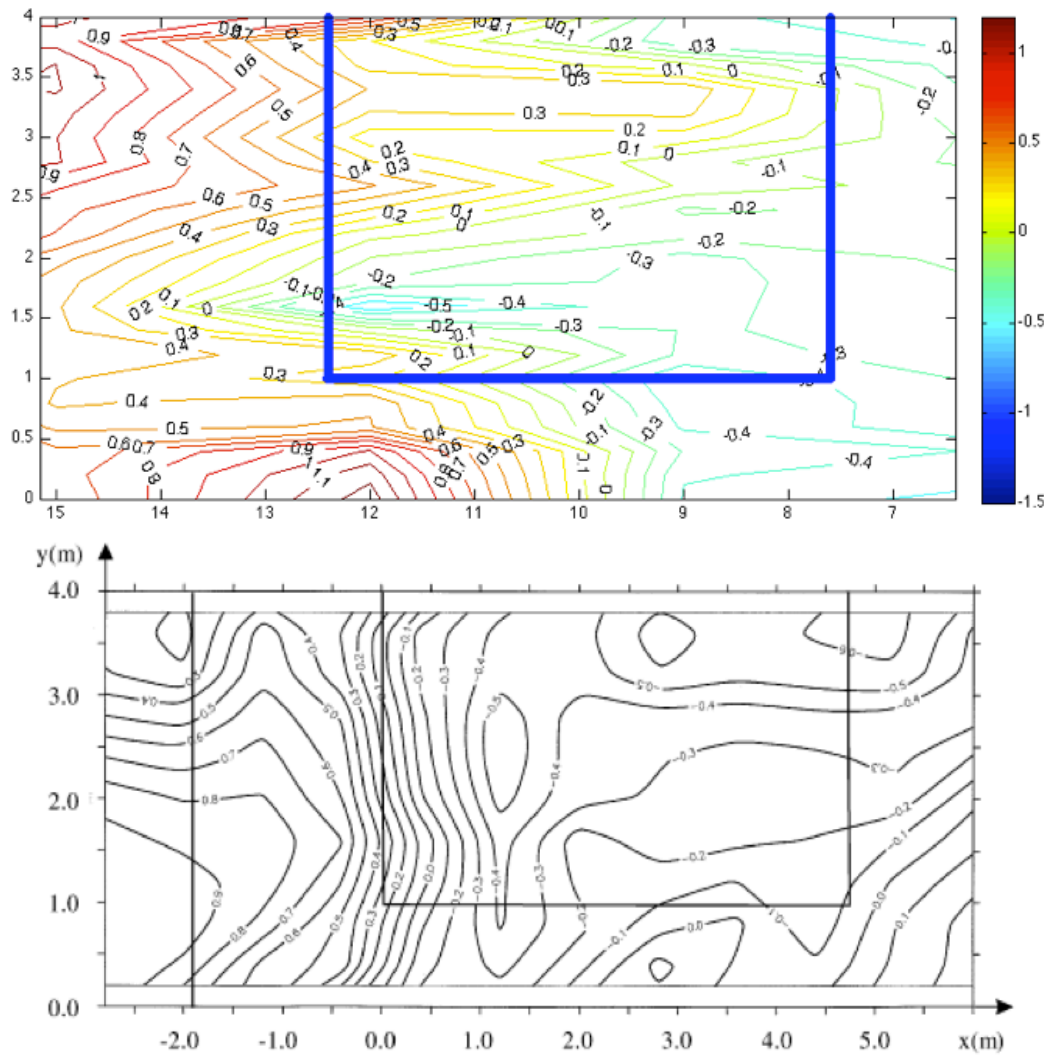

Figure 8. Mean surface elevation in $\mathrm{cm}$. Top: Numerical results. Bottom: Experimental result 
A snapshot of the free surface elevation at time $=40 \mathrm{~s}$ is illustrated in figure (9). Waves are traveling from right to left. The waves start shoaling and breaking on the bar but the waves keep their original shape over the channel until they reach the end of the channel in left hand side. The wave-current interaction can be observed in the channel where the waves are refracted by the current. Waves begin to shoal and break behind the channel in the nearshore area, which lead to the secondary circulation in the surf zone.

In order to study wave-induced circulations and their changes during time, vorticity is calculated and studied in different computational times. Figure (10) shows the mean velocity and vorticity profiles and the unit of color bar for vorticity is $s^{-1}$. In vorticity patterns, blue and red colors denote clock-wise and anti-clockwise rotations respectively. The waves propagate from right to left. The circulation patterns and center of them are given in this figure.
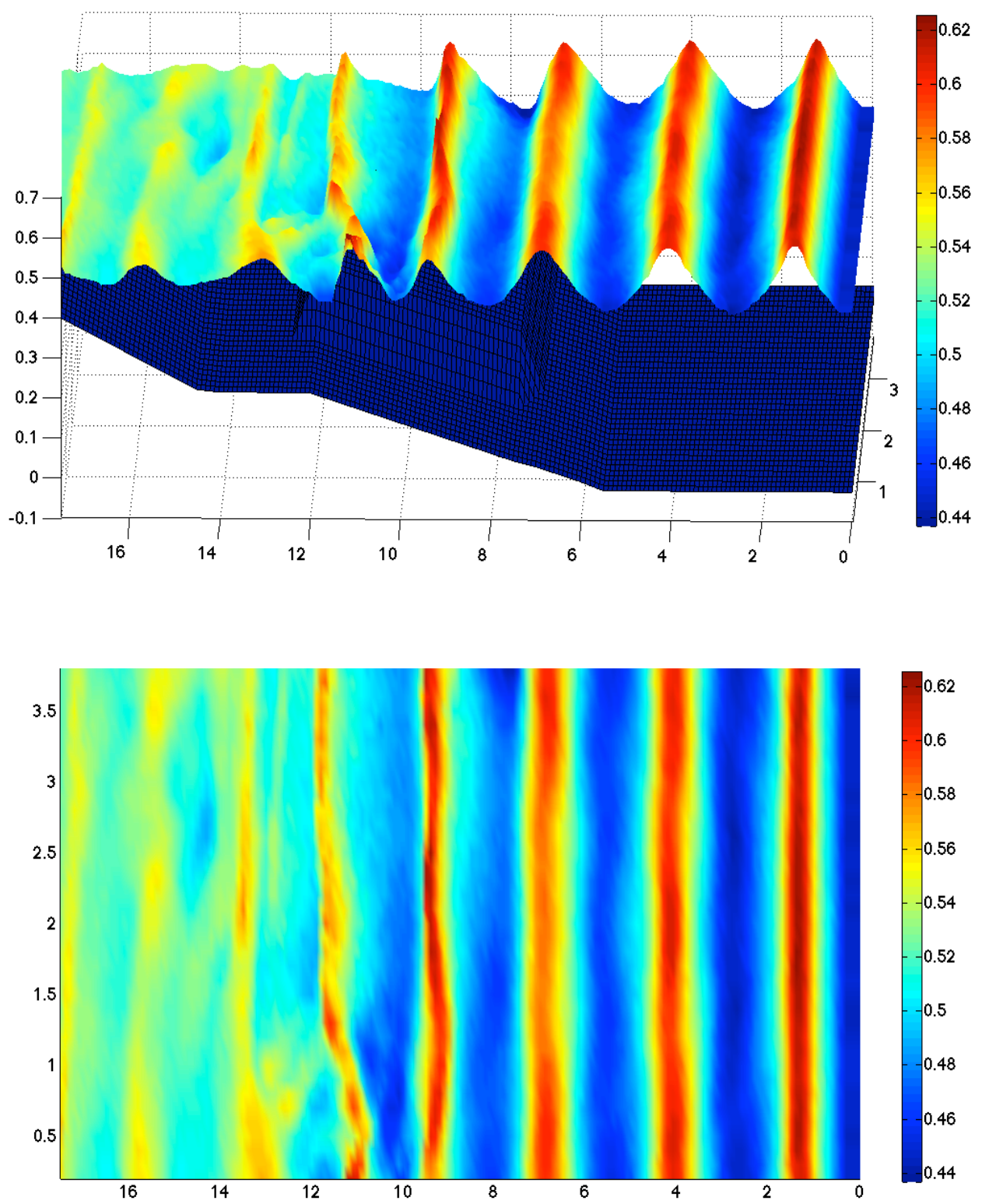

Figure 9. Free Surface Elevation at time $=$ 40-s. Top: Side View. Bottom: Top View

Vorticity is defined as $\Omega_{\alpha}=v_{x}-u_{y}$ where $(u, v)$ are underlying mean current averaged over one wave period. In both of the pictures, two main circulations: one over the bar (rotating anti-clockwise) and one near-shore (rotating clock-wise) are observed but their strength and center of rotation are changing with time. Anti-clockwise vorticity, generated on the crest of the bar, is caused by the long- 
shore non-uniformity of wave breaking patterns on the bar crest. Clockwise vorticity located in surf zone is generated due to larger setup shoreward of the rip channel in comparison with the setup shoreward of the bar. In addition mass flux of wave motion over the bar is much greater than that in rip channel due to wave-current interaction. Two minor circulations: one on the up- right hand side of bar circulation (in the opposite direction of it) and one on the left-down side of near-shore circulation (in the opposite direction of it) can also be observed. Strong vorticity near rip-current sidewall are due to the solid boundary as opposed to a line of symmetry.
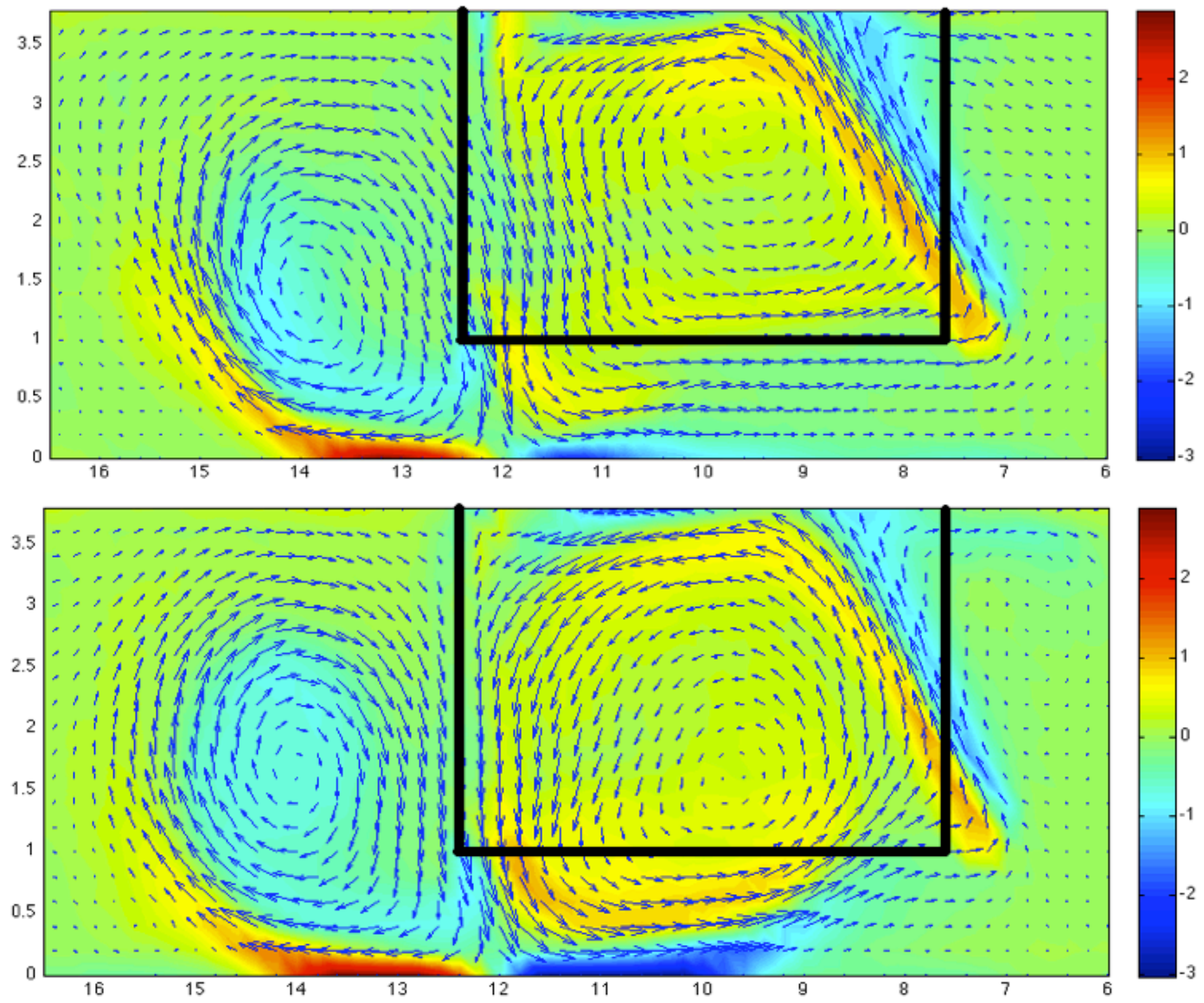

Figure 10. Mean vorticity and mean horizontal velocity distributions in two different times, top: $\mathrm{T}=\mathbf{2 0 - s ,}$ bottom: $\mathrm{T}=40-\mathrm{s}$

\section{Three-Dimensional Variation of Flow Structure}

The three-dimensional distribution of mean horizontal velocity profiles is given in figure (11). In this figure velocities at different points are shown. Velocities have been averaged over one wave period. The figure shows, inshore of the bar, the lateral flow traveling in the along-shore direction from the bar into the rip channel. The lateral flow is non-uniformly distributed in the vertical direction and the reason can be related to the sudden increase in the water depth from the submerged bar into the trough shoreward of the bar. The rip current has a tendency to be stronger closer to the bed in the entrance of the rip channel but the current move toward the surface at offshore end of the channel due to increase in depth (Arthur, 1962). At the entrance of the rip channel, upper part of the water column tries to curve the along-shore flow into the cross-shore current that leads to higher pressure in the upper part of the water column and higher velocities in the lower part. On the bar crest and also the entrance point of rip channel, where trough and rip channel meet, the direction of flow is nonuniform and is dependent on the vertical position, suggesting that three-dimensional effects and rotations are important in the dynamics of rip current system development.

The trajectory of several particles that were initially located in different positions over the bar and in the surf zone at the half water depth, have been tracked as figure (12). It can be observed that some particles over the bar enter the circulation and then flow into the rip channel, while others flow to the surf zone and enter the secondary circulation. The particle that enters the channel tends to go toward 
the surface as it approaches the offshore side of the channel due to wave-current interaction. The particle that was initially in the surf zone seems to stay there and keeps rotating in clock-wise direction.

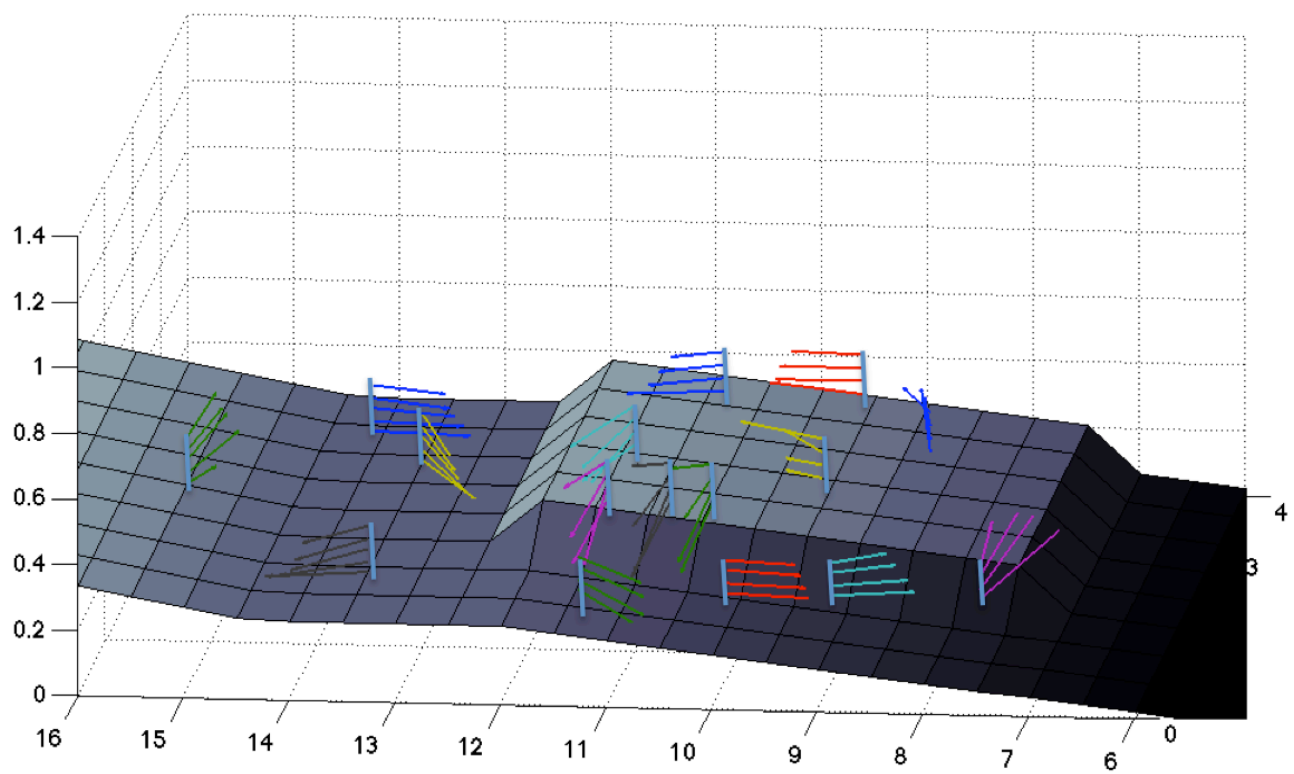

Figure 11. Three dimensional velocity profile

\section{Effect of SPH Particle Resolution}

Different SPH particle spacing is used to model the bar/channel rip current system. Numerical results are compared to test $3 *$ of Drønen experiments where still water depth at the bar crest is equal to $0.1 \mathrm{~m}$ and wave height is equal to $0.15 \mathrm{~m}$. Other information is similar to the previous test. Particle spacing of $0.02 \mathrm{~m}$ (about three million particles), $0.03 \mathrm{~m}$ (about seven hundred thousand particles) and $0.04 \mathrm{~m}$ (about three hundred thousand particles) are used. In all of the cases, circulations can be observed over the bar and in surf zone area but rip current intensity is much lower in the cases with $0.03 \mathrm{~m}$ and $0.04 \mathrm{~m}$ particle spacing. In the case of $0.04 \mathrm{~m}$ particle spacing, almost no rip current happens in the rip channel.

Cross-shore velocities in long-shore direction of rip channel at the position of $x=11.4 \mathrm{~m}$ and one third of the water depth are studied and compared to the experimental results. The mean square errors between the numerical results and experimental results are computed as figure (13). The errors decrease by the decrease of particle spacing until the particle spacing is equal to 0.023 . From then, the mean square errors keep constant with the decrease of the particle spacing so particle spacing equal to 0.023 can be chosen as the convenient amount for this simulation. The numerical results of cross-shore velocity on different positions along the rip channel show a good agreement with the experimental results and the mean least square error is equal to 0.002 .

\section{Conclusion}

A numerical model based on Smoothed Particle Hydrodynamics method is extended to calculate fluid parameters on fixed Eulerian nodes in addition to Lagrangian moving particles. This attempt has been done in order to enhance the ability of three-dimensional numerical method to capture depth-integrated currents as well as three-dimensional structure of flow. Then the model is used to model wave propagation, offshore-directed current and wave-induced nearshore circulation over a rip current system consisting of a bar intersected by a rip channel. The pattern of rip current and two cell circulations, one rotating anti-clockwise over the bar and the other rotating clockwise in the surface zone, is successfully modeled. The non-uniformity of breaking waves over the bar leads to different set-ups and elevation gradients on the submerged bar that force the circulation. As the waves are mostly breaking over the bar and there is almost no breaking in the rip channel, alongshore-directed flow from the bar with higher set-ups into the channel occur that feed the rip current flow. Different wave phenomena such as wave shoaling, wave breaking, diffraction-refraction pattern, wave-current interaction and higher harmonic generation in the surf zone was observed. The model is capable of capturing nearshore nonlinear surface waves as well as underlying rip current flow. Current field and wave-current interactions are investigated by looking at mean horizontal velocities profiles (depth- 
integrated and time-integrated) and vorticity field. Vorticity generation over the bar results from nonuniformity of breaking waves caused by topography perturbations. Three-dimensional structure of flow
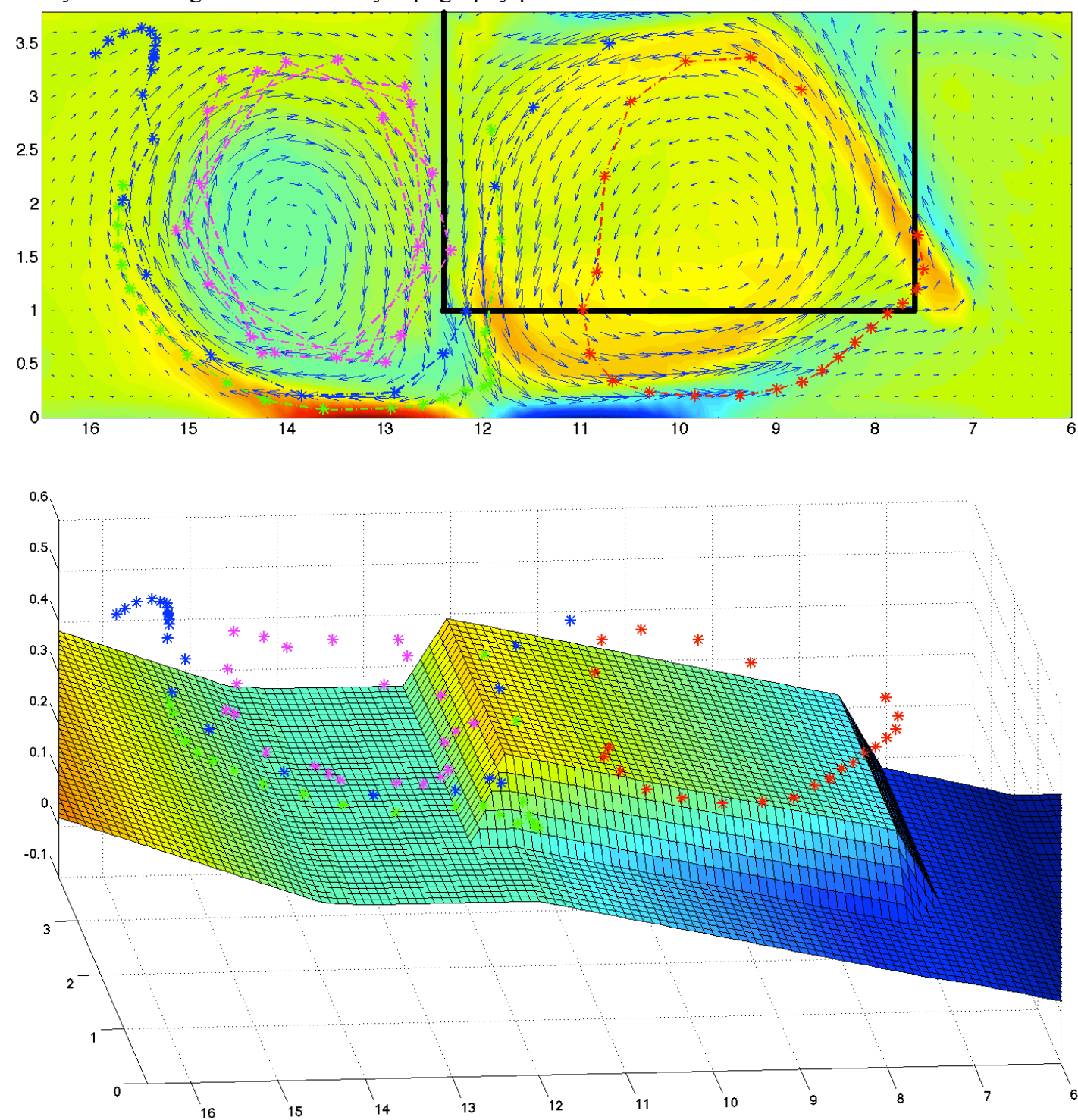

Figure 12. Particle Trajectories. Top: Top view, Bottom: Three-dimensional View

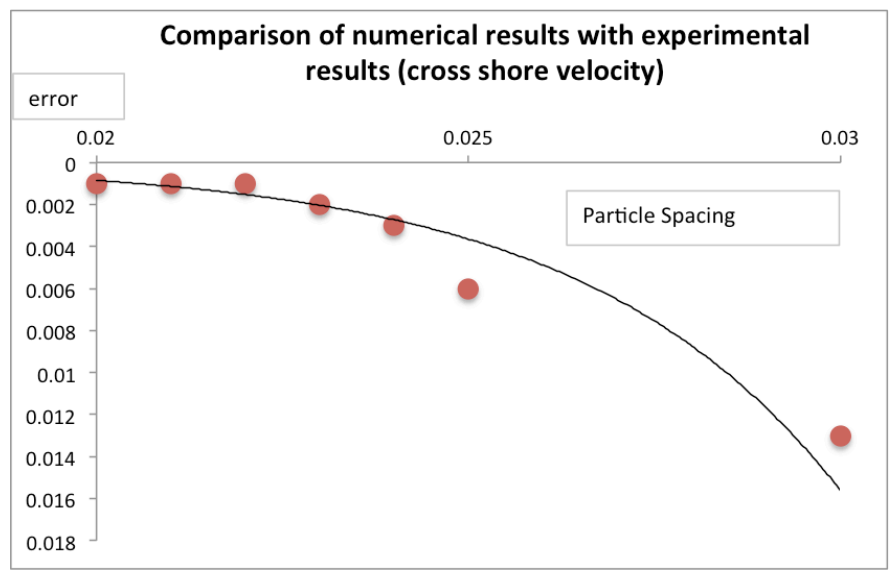

Figure 13. Comparison of numerical results of cross-shore velocity along the rip channel with experimental results 
is studied in different parts of the region. On the bar crest, three-dimensional effects lead to nonuniformity in the flow direction depending on the vertical position. Wave refraction-diffraction caused by underlying rip current, is also studied.

The model results are compared with the observations of laboratory experiments of Drønen et al. (2002) on rip current generated in a region of a single bar and a rip channel. Good agreement is obtained between numerical results and experimental data in terms of mean velocity distribution, mean surface elevation and three-dimensional development of currents.

\section{REFERENCES}

Arthur, R. S., 1962, A note on the dynamics of rip currents, Journal of Geophysical Research, Vol. 67, No. 7, P. 2777-2779.

Bowen A., D. Inman, 1969, Rip currents 1. Theoretical investigation, Journal of Geophysical Research, Vol. 74, No. 23, P. 5467-5478.

Bowen A., Inman D., Rip currents 2. Laboratory and field observations, 1969, Journal of Geophysical research, Vol. 74, No. 23, P. 5479-5490.

Chen Q., R. A. Dalrymple, J. Kirby, A. Kennedy, M. Haller, 1999, Boussinesq modeling of a rip current system, Journal of Geophysical Research, Vol. 104, No. C9, P. 20617-20637.

Colagrosi A., M. Landrini, 2003, Numerical simulation of interfacial flows by smoothed particle hydrodynamics, Journal of Computational Physics, Vol.191, P. 448-475.

Dalrymple, R. A., Knio, O, Cox, D.T., Gómez-Gesteira, M., and Zou, 2002, Using a Lagrangian particle method for deck overtopping, Proceedings of Waves, ASCE. 1082-1091

Dalrymple R. A., B. Rogers, 2006, Numerical modeling of water waves with SPH method, Coastal Engineering, Vol.53, P.141-147.

Dalrymple R. A., J. MacMahan, A. Reniers, V. Nelko, 2011, Rip currents, Annual Review of Fluid Mechanics, Vol.43, P. 551-581.

Drønen N., H. Karunarathna, J. Fredsoe, B. Sumer, R. Deiggard, 2002, An experimental study of rip channel flow, Coastal Engineering, Vol. 45, P. 223-238.

Feldman J., J. Bonet, 2007, Dynamic refinement and boundary contact forces in SPH with applications in fluid flow problems, Journal of Numerical Methods in Engineering, Vol. 27, P. 295-324.

Haller M., R. A. Dalrymple, I. Svendsen, 1997, Rip channels and nearshore circulation: experiments, ASCE, P. 594-603.

Haas K. A., A. Svendsen, 2002, Laboratory measurements of the vertical structure of rip currents, Journal of Geophysical Research, Vol. 107, NO. C5, P. 15-1, 15-19.

Haas K. A., A. Svendsen, M. C. Haller, Q. Zhao, 2003, Quasi-three-dimensional modeling of rip current systems, Journal of Geophysical Research, Vol. 108, NO. C7, P. 10-1, 15-21.

Hérault A., G. Bilotta, R. A. Dalrymple, 2010, SPH on GPU with CUDA, Journal of Hydraulic Research, Vol.48, P. 74-79.

Marrone S., A. Colagrossi, D. Le Touze, G. Graziani, 2010, Fast free-surface detection and level-set function definition in SPH solvers, Journal of Computational Physics, Vol.229, P. 3652-3663.

Mei, C. C., and U. Ünlüata, 1972, Harmonic generation in shallow water waves. Waves on Beaches and Resulting Sediment Transport, P. 181-202.

Monaghan J., 1994, Simulating free surface flows with SPH, Computational Physics, Vol. 110, P. 399406.

Monaghan J., A. Kos, 1999, Solitary waves on a certain beach, Journal of Waterway, Port, Coastal and Ocean Engineering, P.145-154.

Monaghan J., J. Kajtar, 2009, SPH particle boundary forces for arbitrary boundaries, Computer Physics Communications, Vol.180, P. 1811-1820.

Mckenzie P., 1958, Rip current systems, The Journal of Geology, Vol.66, P. 103-113.

Wright L. D., A. D. Short, 1984, Morphodynamic variability of surf zones and beaches: a synthesis, Marine Geology, Vol. 56, P. 93-118.

Panizzo, A., R. A. Dalrymple, 2004, SPH modeling of under-water landslide generated waves. Proc. ICCE, ASCE.

Shepard F. P., K. O. Emery, E. C. La Fond, 1941, Rip currents: A process of geological importance, The Journal of Geology, Vol.49, P. 337-369.

De Vriend, H.J., J. Zyserman, J. Nicholson, J.A. Roelvink, P. Pechon, and H.N. Southgate. 1993. Medium-term 2DH coastal area modeling, Coastal Engineering, 21, 193-224.

Wiegel, R.L. 1965. Oceanographical Engineering, Prentice-Hall, Englewood Cliffs, New Jersey, 531. 